\title{
Feel the force
}

We are
working to ...
expand this
chemistry
to new
photoswitches
with unique
properties

Engineers have long studied the forces acting on macroscopic objects, but only in recent years have chemists begun to apply these principles on a molecular level. These are perhaps best observed in polymers, including archetypal materials in which colourless spiropyran units, when under tension, undergo ring-opening to afford red-purple merocyanines. Such materials enable force to be transduced into an optical readout - but take away the force and each merocyanine relaxes back to its more compact spiropyran form. Writing in Journal of the American

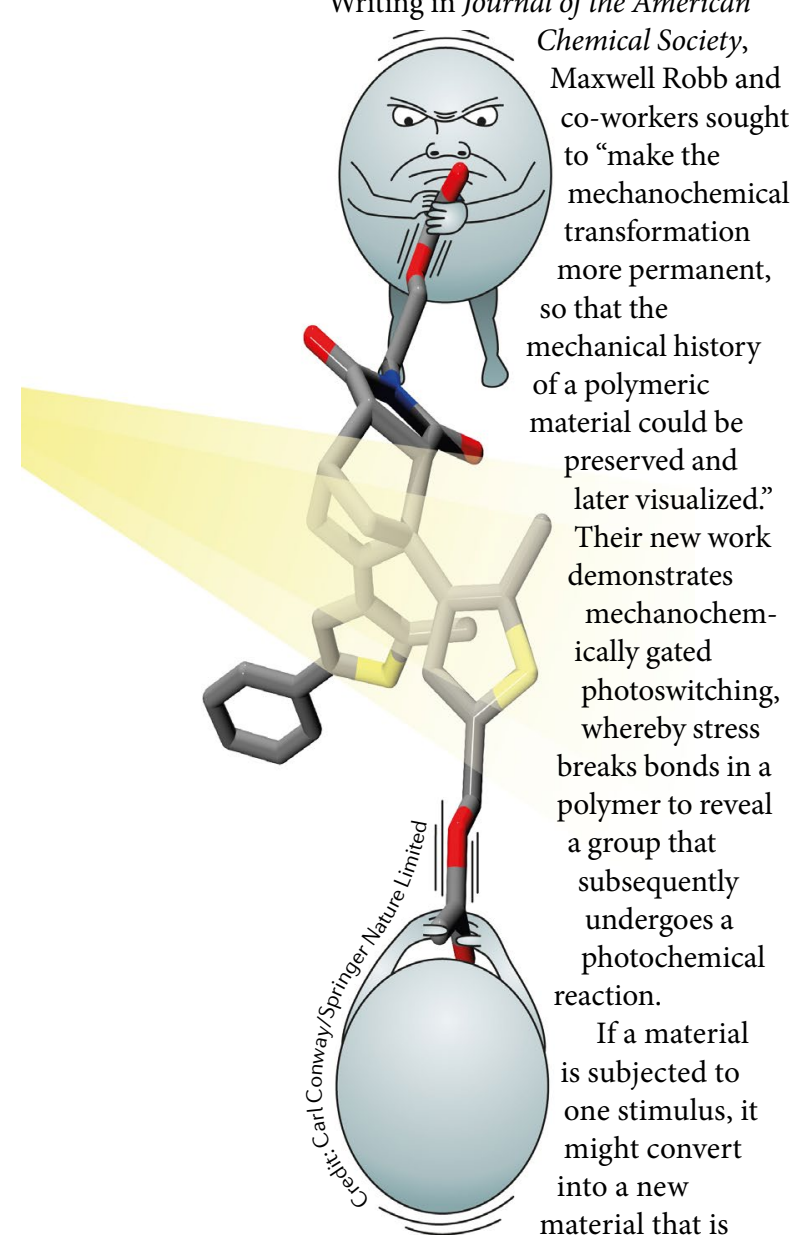

only then susceptible to a second stimulus. This principle is known for mechanical gating of mechanochemistry, and reactivity gating of diarylethene photoswitching. Robb's team combined these two concepts to design a new approach in which a force-sensitive group known as a mechanophore - is converted into a light-sensitive group. The latter took the form of a functionalized 1,2-bis(3thiophenyl)cyclopentadiene that can undergo UV-light-triggered electrocyclization, a reaction signalled by the formation of a red cyclohexadiene product. However, this reaction cannot occur if the cyclopentadiene is trapped with an alkene as part of a Diels-Alder adduct. Such a norbornene is photochemically inert but mechanically sensitive because it can dissociate under stress through a retro-Diels-Alder reaction. The most promising design was an adduct between a 1,2-bis(3thiophenyl)cyclopentadiene and a maleimide. Indeed, density functional theory calculations indicate that if the molecule pictured is stretched from its equilibrium geometry by just over $3 \AA$, it experiences enough force to break into its two constituents. Comparing the rupture force to calculations of other known mechanophores, it seemed reasonable that the reaction could be reproduced in a laboratory.

Robb's team took their DielsAlder adduct and decorated the maleimide and one thiophene each with a bromoisobutyryl ester - a radical polymerization initiator from which poly(methyl acrylate) chains were grown. The norbornene was now in the middle of $\sim 90 \mathrm{kDa}$ of poly(methyl acrylate) and susceptible to forces transmitted through its two long arms. These forces were applied using solutionphase ultrasonication, whereby cavitation leads to stresses that are greatest near the centre of a polymer chain. Thus, the norbornene groups ruptured to afford maleimide and 1,2-bis(3thiophenyl)cyclopentadiene units, both of which featured similar poly(methyl acrylate) arms. The mixture remained colourless after ultrasonication, and it was only upon subsequent UV irradiation that electrocyclization of the 1,2-bis(3-thiophenyl) cyclopentadiene gave the corresponding red cyclohexadiene - an encouraging proofof-concept of the new stress sensor.

The gating observed by Robb and his colleagues opens many possibilities, including singlemolecule force measurements. Moreover, one could envisage a polymer in which loops are each cinched with a mechanophore, the cleavage of which still leaves the polymer intact. "We are working to streamline the synthesis, expand this chemistry to new photoswitches with unique properties and also modulate the mechanochemical activity," shared Robb. Once these aspects are addressed, the responsive materials may well find new applications in patterning, data storage and stress sensing.

David Schilter

ORIGINAL ARTICLE Hu, X., McFadden, M. E., Barber, R. W. \& Robb, M. J. Mechanochemical regulation of a photochemical reaction. J.Am.Chem. Soc. https://doi.org/10.1021/ jacs.8b09628 (2018) 